\title{
Acute Cervical facet Joint Inflammation After a Prolonged Dental Procedure: A Case Report
}

\author{
Lucas First, MD ${ }^{1}$ \\ Michael Sein, MD²
}

\begin{abstract}
Background: Facet joints of the spine are the only true synovial joints between adjacent spinal levels. Degenerative disease changes of the joint, or facet arthropathy, can be asymptomatic. However, pain can occur when the joint becomes acutely inflamed. Specifically, cervical facet arthropathy and associated neck pain can be difficult to treat.

Case Report: We present a case of a 64-year-old man with known lumbar spondylosis who developed acute neck pain after a dental procedure. Magnetic resonance imaging (MRI) revealed inflammation of the left C2-C3 facet joint. This patient achieved successful relief of pain with an intraarticular corticosteroid injection followed by physical therapy. Careful consideration was given to a detailed therapy protocol in order to regain pain-free active range of motion of the neck.

Conclusion: To our knowledge, this is the first reported case of acute facet inflammation after a dental procedure. Additionally, this report highlights the value of intraarticular corticosteroids for an acutely inflamed facet joint. In order to prevent injury, dentists and oral surgeons should be attentive to neutral spine positioning, especially during prolonged procedures.
\end{abstract}

Key words: Arthritis, cervical spine, corticosteroids, dentistry, facet joint, neck pain, physical therapy, posture

\section{BACKGROUND}

Cervical facet arthropathy is one of the most common causes of neck pain (1). Neck pain is associated with significant disability in the general population, and chronic neck pain is seen in up to $60 \%$ of patients after the initial pain episode (2). Facet joints are innervated by mechanoreceptors and nociceptors, with disruption to the facet capsule a main pain generator $(1,3)$. The cervical facet capsular ligament is vulnerable to excessive stretch, and injury occurs with neck extension loading, which can alter the biochemical and mechanical microenvironment of the joint (3). Referred pain patterns from each facet level of the cervical spine have been well described. In general, $\mathrm{C} 1-\mathrm{C} 2$ and $\mathrm{C} 2-\mathrm{C} 3$ joints result in suboccipital pain, the $\mathrm{C} 3-\mathrm{C} 4$ joint produces posterolateral neck pain in the area of the levator scapulae muscle, the C4-C5 joint involves the junction of the inferior neck and upper limb girdle overlying the upper trapezius muscle, and the $\mathrm{C} 5-\mathrm{C} 6$ and $\mathrm{C} 6-\mathrm{C} 7$ joints extend laterally to the

From: ${ }^{1}$ New York Presbyterian Hospital, New York, NY; ${ }^{2}$ University of Florida Health, Gainesville, FL

Corresponding Author: Lucas First, MD, E-mail: Iff9006@nyp.org

Disclaimer: There was no external funding in the preparation of this manuscript.

Conflict of interest: Each author certifies that he or she, or a member of his or her immediate family, has no commercial association (i.e., consultancies, stock ownership, equity interest, patent/licensing arrangements, etc.) that might pose a conflict of interest in connection with the submitted manuscript. 
acromioclavicular joint and posteriorly over the scapula (4). During dental procedures, patients may be subjected to prolonged periods of neck extension. There are previous reports of pyogenic cervical facet joint infections and neuropathic pain syndromes after dental procedures; however, to our knowledge, acute facet inflammation as a result of neck positioning has not been previously described $(5,6)$. We present a case of acute neck pain after a dental procedure.

\section{CASE PRESENTATION}

The patient is a 64-year-old man who presented to a comprehensive spine center with a chief complaint of acute left-sided neck pain following a dental procedure. History was significant for spondylosis of his lumbar spine. His new neck pain began approximately 24 hours after a prolonged dental procedure in hyperextension and his initial clinic evaluation was one week after the symptoms began. The pain was described as sharp in nature and located in the suboccipital region. He denied numbness, tingling, and weakness in his upper extremities.

Physical exam was pertinent for tenderness to palpation of the facet

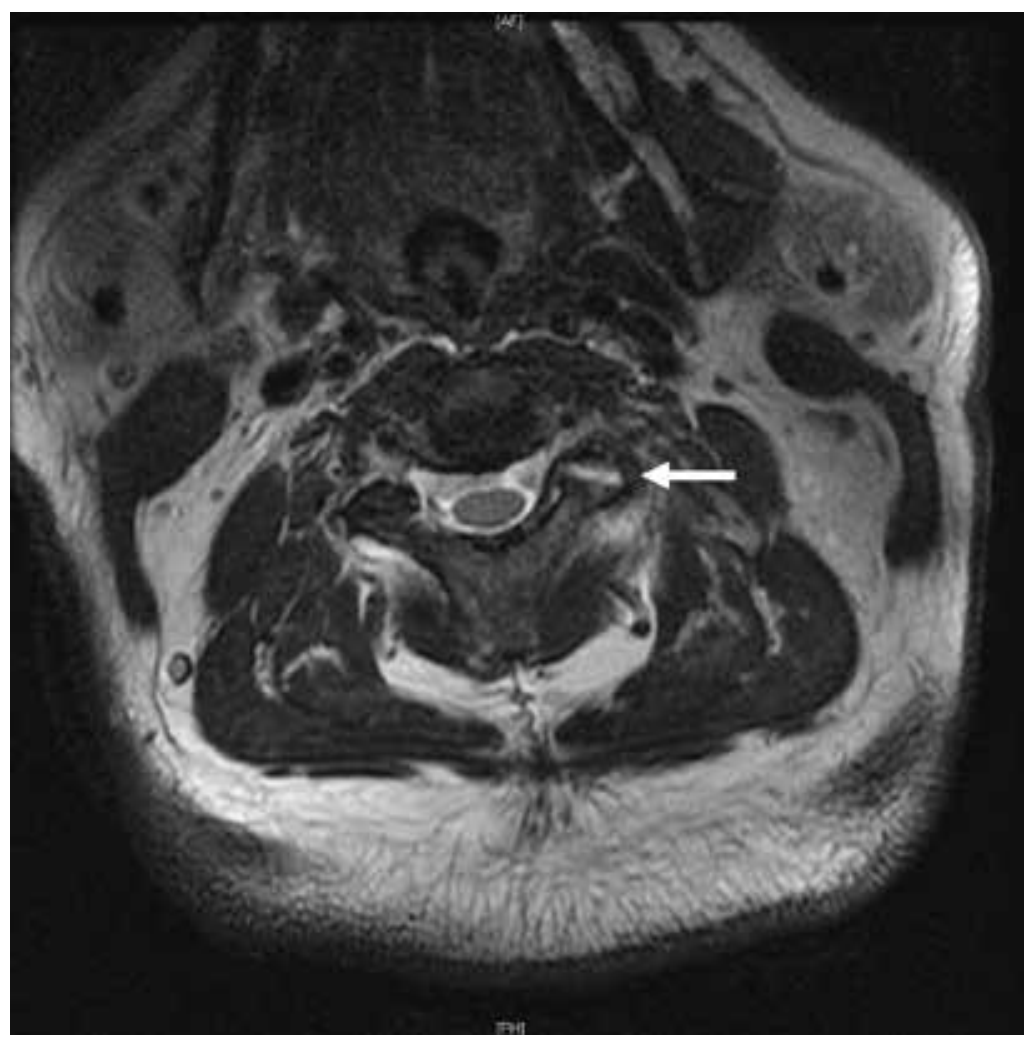

Fig. 1. T2-weighted, axial plane, cervical MRI - demonstrating hyper-intensity in the left C2-3 facet joint likely representing edema (white arrow). This correlated to the patient's side and distribution of symptoms. columns, and increased pain with neck extension and facet loading on the left. Spurling's maneuver was negative and the patient was neurologically intact. He was prescribed a brief course of methylprednisolone. His symptoms persisted and a noncontrast-enhanced cervical spine MRI was obtained.

Imaging revealed marrow edema surrounding the left $\mathrm{C2}-\mathrm{C} 3$ facet joint with underlying uncovertebral and facet hypertrophy (Fig. 1). Other findings included straightening of the cervical spine and diffuse disc desiccation with the greatest loss of disc height and endplate degenerative changes at $\mathrm{C} 5-\mathrm{C} 6$ and $\mathrm{C6}-\mathrm{C} 7$. The radiologist summarized the findings as multilevel spondylosis superimposed on congenital narrowing of the canal, most notable for moderate C4-C5 and moderate-to-severe C5-C6 canal stenosis. Moderate bilateral $\mathrm{C} 4-\mathrm{C} 5$ and severe right $\mathrm{C} 6-\mathrm{C} 7$ foraminal narrowing were also noted. No abnormal cord signal was visualized. Given the patient's continued symptoms, exam findings, and imaging results, he underwent a fluoroscopically guided injection of the left $\mathrm{C} 2-\mathrm{C} 3$ facet joint with corticosteroid and local anesthetic. The procedure was performed about 2 and a half weeks after his initial presentation.

For the procedure, the patient was placed in a lateral decubitus position with the left side up and Universal Protocol was utilized. The lateral approach was preferred, as the more cephalad cervical segments, in this case $\mathrm{C} 2-\mathrm{C} 3$, are easily accessible without concern that shoulders would obscure the target. All options, risks, and benefits were discussed with the patient prior to the procedure. Skin was anesthetized with one $\mathrm{mL}$ of $1 \%$ lidocaine and then a 25-gauge, 3.5-inch needle was directed toward the target using intermittent fluoroscopic guidance. A small amount of contrast dye was injected without 
evidence of vascular uptake (Fig. 2). Finally, the facet joint was injected with one $\mathrm{mL}$ of methylprednisolone at $40 \mathrm{mg} / \mathrm{mL}$. The patient tolerated the procedure well, without complications.

The patient reported immediate postprocedure pain relief. He was referred to physical therapy focused on neuromuscular control of cervical and scapular retraction, while maintaining neutral spine positioning with initial avoidance of end-range cervical extension. He progressed to isometric cervical flexion-strengthening exercises. Good posture and body mechanics were emphasized. Restoration of baseline pain-free cervical active range of motion was achieved. To date, the patient has not returned to the clinic with a reoccurrence of symptoms.

\section{DISCUSSION}

Facet joints of the spine are the only true synovial joints between adjacent spinal levels, and studies have shown the prevalence of asymptomatic cervical facet arthropathy to be as high as 33\% $(7,8)$. Symptoms may develop after an inciting event such as a whiplash injury. Additionally, mechanical stress placed on the facet joints with prolonged neck extension may potentiate symptoms. About $65 \%$ of all adults 18 years and older in the United States visit the dentist at least once per year (9), with a majority of procedures occurring with the patient in neck extension. Therefore, in patients with a history of spondylosis, dentists and oral surgeons should be aware of proper neutral spine positioning to prevent acute inflammation of cervical facet joints. If not technically feasible, an effort should be made to limit the time spent in hyperextension.

Treatment paradigms for cervical facet arthropathy have been established. Controversy still exists surrounding the various treatments utilized. Systematic reviews have shown strong evidence for the diagnostic accuracy of local anesthetic blocks to the joint, which may lead to radiofrequency neurotomy; however, the evidence for the use of therapeutic intraarticular injections is weaker (10). Corticosteroids possess anti-inflammatory and antiedema properties, which are beneficial when the synovium of the facet joint is inflamed. Joint inflammation likely results in pain due to the abundance of nociceptive C-fibers of the synovial lining (11). A study by Lim et al (11) found that intraarticular corticosteroid injections into cervical facet joints resulted in significant pain relief at 1, 3, and 6 months after the procedure.

\section{CONCLUSION}

In this report, we discuss a case of acute unilateral, unilevel facet arthropathy following a prolonged dental procedure with symptoms resolving rapidly after intraarticular corticosteroid injection. When evaluating patients with acute-onset neck pain, history of recent dental procedures may be helpful in identifying patients

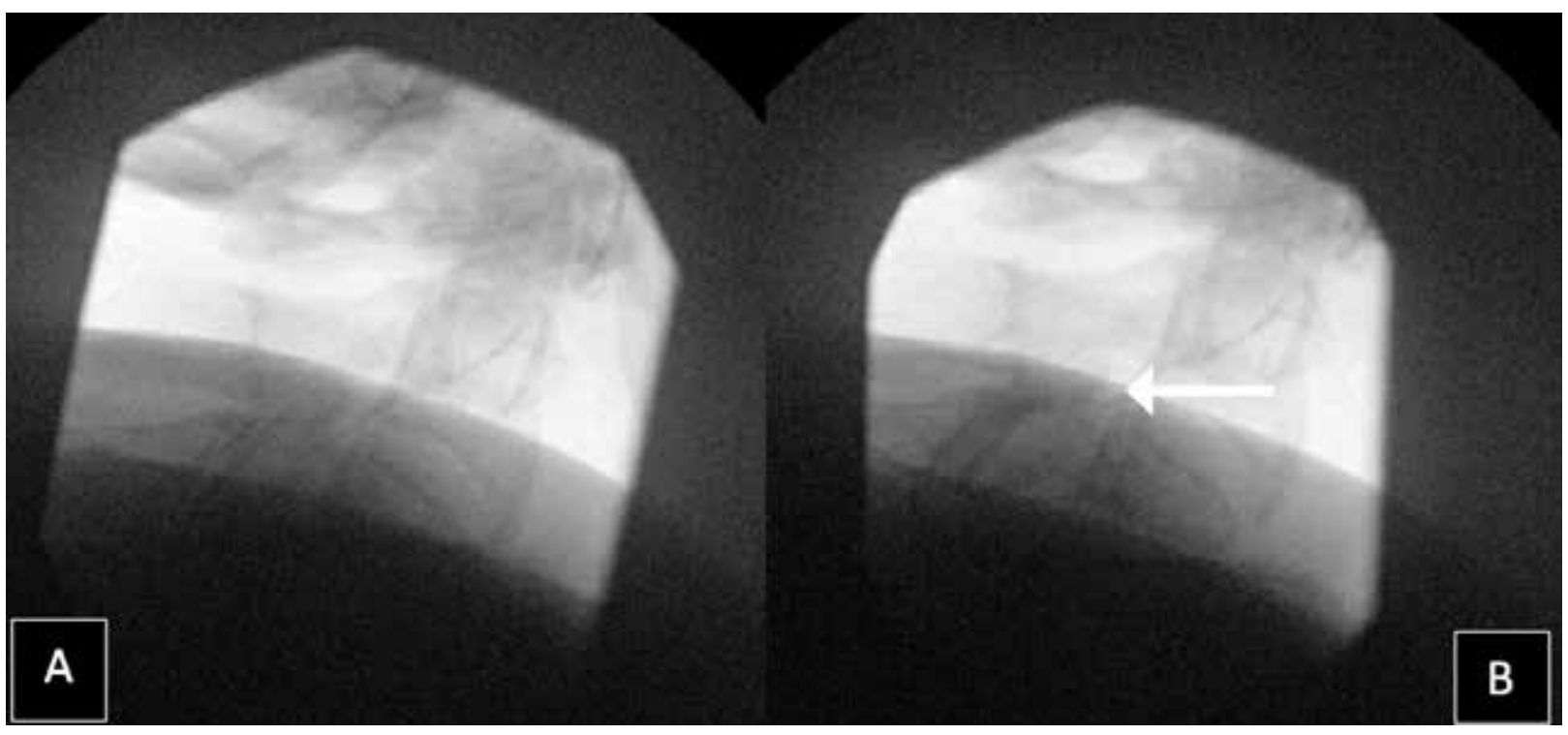

Fig. 2. Fluoroscopy images demonstrating before $(A)$ and after $(B)$ contrast administration with faint intra-articular contrast flow at the C2-C3 facet joint (white arrow). 
with facet-mediated pain. In patients with a history of spondylosis, dental and oral health professionals should be mindful of head and neck positioning, which may cause an acute exacerbation of these conditions.
Additionally, in such cases, early treatment with intraarticular corticosteroid injections may obviate the need for medial branch blocks and ablation with radiofrequency.

\section{REFERENCES}

1. Manchikanti L, Singh V, Falco F, Cash K, Fellows B. Cervical medial branch blocks for chronic cervical facet joint pain: A randomized, double-blind, controlled trial with one-year follow-up. Spine 2008; 33:1813-1820

2. Côté $P, C$ assidy D, Carroll L, Kristman V. The annual incidence and course of neck pain in the general population: A population-based cohort study. Pain 2004; 112:267-273.

3. Sperry MM, Ita ME, Kartha S, Zhang S, Yu Y-H, Winkelstein B. The interface of mechanics and nociception in joint pathophysiology: Insights from the facet and temporomandibular joints. J Biomech Eng 2017; 139:02100301-02100313.

4. Cooper G, Bailey B, Bogduk N. Cervical zygapophysial joint pain maps. Pain Med 2007; 8:344-353.

5. Kaye ID, Protopsaltis TS. Cervical facet joint infection and associated epidural abscess with streptococcus intermedius from a dental infection origin: A case report and review. Bull Hosp Jt Dis 2016; 74:237-243.
6. Tinastepe N, Oral K. Neuropathic pain after dental treatment. Agri 2013; 25:1-6.

7. Kim JH, Sharan A, Cho W, Emam M, Hagen M, Kim SY. The prevalence of asymptomatic cervical and lumbar facet arthropathy: A computed tomography study. Asian Spine J 2019; 13:417-422.

8. Gellhorn AC, Katz JN, Suri P. Osteoarthritis of the spine: The facet joints. Nat Rev Rheumatol 2013; 9:216-224.

9. Oral and Dental Health. Centers for Disease Control and Prevention Web site. www.cdc.gov/nchs/fastats/dental.htm. Date Accessed 06/02/2019.

10. Falco FJE, Manchikanti L, Datta S, et al. Systematic review of the therapeutic effectiveness of cervical facet joint interventions: An update. Pain Physician 2012; 15:E839-E868.

11. Lim JW, Cho YW, Lee DG, Chang MC. Comparison of intraarticular pulsed radiofrequency and intraarticular corticosteroid injection for management of cervical facet joint pain. Pain Physician 2017; 20:E961-E967 